\title{
Registrierung von Studien in öffentlich zugänglichen Registern: Stand der Dinge
}

\section{Myriam Rège-Walther, \\ Bernard Burnand}

Cochrane Schweiz Institut universitaire de médecine sociale et préventive (IUMSP), CHUV et Université de Lausanne

Wir danken Dr. med. Erik von Elm für das Korrekturlesen der deutschen Version.
Korrespondenz:

Myriam Rège-Walther

Unité d'évaluation des soins

Institut universitaire

de médecine sociale et préventive (IUMSP)

CHUV et Université

de Lausanne

Bugnon 17

CH-1005 Lausanne

myriam.rege@chuv.ch

www.swiss.cochrane.org

\section{Die Situation}

Mehr Transparenz, bitte! Dies fordern immer mehr Menschen, öffentliche Organisationen und Institutionen, welche die Ergebnisse der medizinischen Forschung für ihre Entscheidungsfindung im Pflegebereich verwenden. Diesem Verlangen nach mehr Transparenz ging die Erkenntnis voraus, dass Ergebnisse klinischer Studien der Pharmaindustrie manipuliert, verschleiert oder nur teilweise offengelegt worden waren - mit unmittelbaren Folgen für die Gesundheit sowie die Kosten einzelner Patienten und öffentlich-rechtlicher Körperschaften. Dabei hätte durch die Veröffentlichung aller Studienergebnisse die Sicherheit der betroffenen Patienten gewährleistet werden können. Die jüngste, vom British Medical Journal (BMJ) angestossene Polemik über die tatsächliche Wirksamkeit von Oseltamivir (Tamiflu ${ }^{\circledR}$ ) zur Verhinderung von Grippekomplikationen verdeutlicht diese Problematik sehr gut [1]. So wurden acht von Roche durchgeführte, unveröffentlichte klinische Studien in eine systematische Review aufgenommen, die zu dem Schluss kam, dass Tamiflu für die Reduzierung grippebedingter Komplikationen bei Erwachsenen wirksam ist [2]. Die Ergebnisse dieser acht Studien waren jedoch nicht bei der Aktualisierung einer Cochrane-Review verfügbar, die die Wirksamkeit von Neuraminidase-Hemmern für die Prävention und Therapie von Grippeerkrankungen bei normalerweise gesunden Erwachsenen untersuchte [3]. Ohne Berücksichtigung dieser acht Studien gelangte die Cochrane-Review zu der Schlussfolgerung, dass die Wirksamkeit von Tamiflu zur Verhinderung grippebedingter Komplikationen bei gesunden Erwachsenen nicht ausreichend nachgewiesen werden kann. Angesichts dieser abweichenden Ergebnisse und der fehlenden Transparenz von Roche stellt sich so weiterhin die Frage, ob Tamiflu grippebedingte Komplikationen bei Erwachsenen wirksam verhindern kann.

In den vergangenen zehn Jahren wurde dieses Problem in mehreren Initiativen angegangen und versucht, die Transparenz in der klinischen Forschung zu verbessern. Besonders die Weltgesundheitsorganisation (WHO), das International Committee of Medical Journal Editors (ICMJE) und der Weltärztebund (WMA) rufen Forscher auf, ihre klinischen Studien in einem öffentlich zugänglichen Verzeichnis zu registrieren und über die Ergebnisse vollständig und innerhalb angemessener Veröffentlichungsfristen zu berichten. Durch eine solche Registrierung können in der Tat systematische Fehler (Bias) identifiziert und verhindert werden, die durch unvollständige oder unterlassene Publikation von Studienergebnissen entstehen. Darüber hinaus kann herausgefunden werden, welche Studien durchgeführt werden müssen, um die bereits vorhandenen Studienergebnisse zu ergänzen. Die klinische Forschung kann so besser geplant, Prioritäten können gesetzt und Doppelspurigkeiten vermieden werden. Betont werden muss hier auch die ethische Notwendigkeit von Transparenz gegenüber den Patienten, die sich freiwillig und ohne eigenen Nutzen für Studien zur Verfügung stellen und erwarten können, dass die Ergebnisse der Studien, an denen sie beteiligt waren, veröffentlicht werden.

Bereits 2005 hat daher die WHO die International Clinical Trials Registry Platform (ICTRP) lanciert [4]. Dieses System dient der leichteren Erfassung klinischer Studien und Veröffentlichung einer Minimalbeschreibung gemäss einer von der WHO entwickelten Liste. Eine Online-Plattform ermöglicht die Suche nach klinischen Studien [5], die in den beteiligten Primärregistern geführt werden und spezifischen Kriterien der WHO genügen. Dieses System zur Studienregistrierung basiert auf Freiwilligkeit und ist rechtlich nicht bindend.

Seit 2007 fordert das Comité International des Editeurs de Journaux Médicaux (ICMJE [6]), dass alle klinischen Studien, die in einer angehörenden Fachzeitschrift veröffentlicht werden sollen, vor der Rekrutierung des ersten Patienten in einem öffentlich zugänglichen Register eingetragen werden. Diese Anregung bindet jedoch nur diejenigen Forscher, die ihre Studienergebnisse überhaupt veröffentlichen wollen. Das ist jedoch nicht bei allen der Fall.

Der Weltärztebund (WMA) hat als Autor der Deklaration von Helsinki, die sich mit jenen ethischen Grundsätzen befasst, die in der medizinischen Forschung am Menschen Anwendung finden, im Jahr 2008 Grundsatz 19 der Deklaration wie folgt revidiert: «Jede klinische Studie ist vor der Rekrutierung der ersten Versuchsperson in einer öffentlich zugänglichen Datenbank zu registrieren.» [7]. Auch hier handelt es sich keinesfalls um eine rechtsverbindliche Verpflichtung, sondern vielmehr um eine ethische Empfehlung.

Diese Initiativen waren relativ erfolgreich. Bis Anfang 2009 erfüllten über 20000 klinische Studien die ICMJE-Anforderungen [8], während es im Jahr 2004 erst 3000 waren.

Dennoch erscheint ein Grossteil der zum Wirksamkeitsnachweis durchgeführten klinischen Studien nie oder nur teilweise in den öffentlich zugänglichen 
Es sollte nicht möglich sein, unerwünschte Studienergebnisse zu verschweigen.

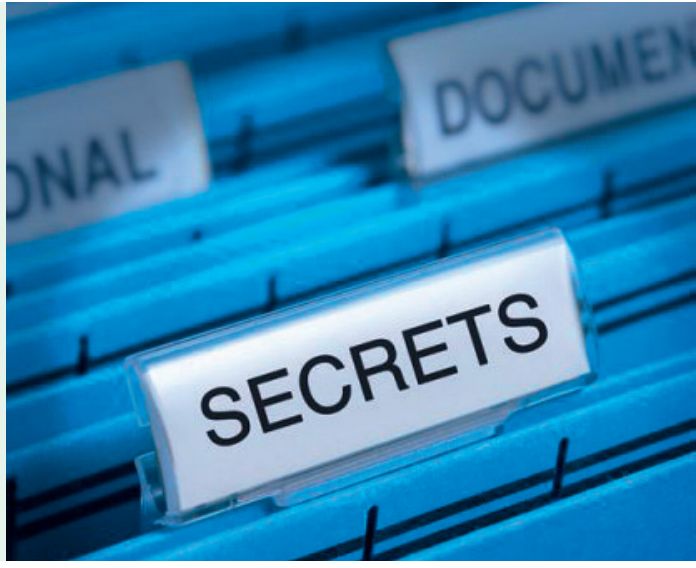

Registern. Daher lässt sich nicht sagen, wie viele klinische Studien weltweit durchgeführt werden, wie gross der Anteil von Studien ist, zu der die Öffentlichkeit keinen Zugang hat, und welche Forschungsfragen darin aufgegriffen werden.

\section{Was ist zu tun?}

Lösungen? Einerseits könnte der Anteil der öffentlichen Forschungsmittel erhöht werden, um so auch diejenigen Interventionen zu untersuchen, an denen die Privatwirtschaft nur wenig Interesse hat. Zum anderen könnten Gesetze erlassen werden, die die Sponsoren verpflichten, Studienprotokolle, laufende Studien und ihre Ergebnisse in frei zugänglichen Registern in verständlicher Form und unter Erfüllung von Mindestanforderungen (wie jenen, die von der WHO empfohlen werden) zu veröffentlichen. Diese beiden Optionen trügen zu einer Erhöhung der Transparenz bei.

Das geltende Schweizer Recht schreibt die Registrierung klinischer Studien in öffentlich zugänglichen Registern nicht vor. Swissmedic, die für die Zulassung, die Herstellungs- und Qualitätskontrolle sowie die Aufsicht von therapeutischen Produkten zuständige Behörde des Eidgenössischen Departements des Innern (EDI), verfügt über ein Register klinischer Medikamentenstudien, das für die Öffentlichkeit nicht zugänglich ist.

Dennoch gibt es Grund für Optimismus. Am 7. März 2010 hat das Schweizervolk dem Art. 118b der Verfassung zugestimmt. Dieser bildet die verfassungsmässige Grundlage für das Bundesgesetz über Humanforschung. Art. 55 des Gesetzentwurfs [9] erwähnt ausdrücklich, dass die Eidgenossenschaft ein öffentliches Register für genehmigte Forschungsprojekte und Zusammenfassungen von Studienergebnissen führt und dass der Bundesrat Bestimmungen hinsichtlich der Daten erlässt, die in diesem Register ausgewiesen sein müssen.

Vor kurzem, am 10. März 2011, hat der Nationalrat das Humanforschungsgesetz als Ganzes angenommen. Artikel 55 wurde aber geändert und der Ausdruck «bewilligte Forschungsprojekte» wurde durch «interventionelle klinische Studien» ersetzt und beschränkt.

Ende März hat sich auch die Kommission für Wissenschaft, Bildung und Kultur des Ständerates mit der
Vorlage befasst; die Abstimmung darüber wurde jedoch vertagt. «Bei der Registrierungspflicht von Forschungsprojekten (Art. 55) nahm die Kommission mit 12 gegen 0 Stimmen eine eigene Variante an, welche diese Pflicht festschreibt, dem Bundesrat aber die Möglichkeit gibt, in Anlehnung an anerkannte internationale Regelungen Ausnahmen davon zu bezeichnen.» [9]

Nun ist der Ständerat am Ball und wird über dieses Gesetzprojekt wahrscheinlich während der Sommersession 2011 beraten.

Am Ende dieser parlamentarischen Debatten und in einer näheren Zukunft wird unser Land vielleicht über eine Gesetzgebung verfügen, die den Zugang zu validen Informationen über abgeschlossene, laufende und künftige klinische Studien garantiert. Ärzte, Institutionen und Patienten könnten so ein wertvolles Hilfsmittel erhalten, um ihre die Gesundheit betreffenden Entscheidungen auf eine bessere Kenntnis der Fakten stützen zu können.

\section{Referenzen}

1 Cohen D. Complications: tracking down the data on oseltamivir. BMJ. 2009;339:b5387.

2 Kaiser L, Wat C, Mills T, Mahoney P, Ward P, Hayden F. Impact of oseltamivir treatment on influenza-related lower respiratory tract complications and hospitalizations. Arch Intern Med. 2003;163:1667-72.

3 Jefferson T, Jones MA, Doshi P, Del Mar CB, Dooley L, Hama R, Heneghan CJ. Neuraminidase inhibitors for preventing and treating influenza in healthy adults. Cochrane Database of Systematic Reviews. 2006; Issue 3. Art. No.: CD001265. Doi: 10.1002/14651858. CD001265.pub2.

4 WHO; international clinical trials registry platform, search portal, http://apps.who.int/trialsearch/ (Webseite im Dezember 2010 besucht).

5 Definition der klinischen Studie gemäss WHO: Im Hinblick auf die Studienregistrierung ist eine klinische Studie jede Art von Forschung, bei der Teilnehmer oder Teilnehmergruppen von Beginn an einer bestimmten Intervention zugeteilt werden, um die Wirkung dieser Intervention auf ihre Gesundheit zu bewerten. Klinische Studien werden auch «interventionelle Studien» genannt. Interventionen können u. a. Medikamente, Zellen und andere biologische Produkte, chirurgische Eingriffe, radiologische Techniken, bestimmte Vorrichtungen, Verhaltenstherapien, Änderungen von Behandlungsprotokollen, Präventionsmassnahmen sein. Diese Definition schliesst Studien der Phasen I bis IV ein.

6 International Committee of Medical Journal Editors. www.icmje.org/ (Webseite im Dezember 2010 besucht).

7 Weltärztebund (WMA). Deklaration von Helsinki des WMA - Ethische Grundsätze im Zusammenhang mit der medizinischen Forschung am Menschen. www.wma.net/fr/30publications/10policies/b3/ index.html (Webseite im Dezember 2010 besucht).

8 Ghersi D, Pang T. From Mexico to Mali: four years in the history of clinical trial registration. Journal of Evidence-Based Medicine. 2009;2:1-7. doi: 10.1111/j.1756-5391.2009.01014.x

9 Medienmitteilung WBK-S: Forschung am Menschen. Bundesgesetz: Ständeratskommission auf Bundesratslinie. 